\title{
Utilização de tecnologias para educação em saúde na Atenção Primária: revisão integrativa da literatura
}

\author{
Use of technologies for health education in Primary Care: integrative literature review \\ Uso de tecnologías para la educación sanitaria en Atención Primaria: revisión integradora de la
}

Yanka Leticia Amorim Uchoa

ORCID: https://orcid.org/0000-0003-4822-5834 Universidade do Estado do Pará, Brasil E-mail: yankaamorym@gmail.com

Andreza Alves Pessôa

ORCID: https://orcid.org/0000-0003-3486-8919 Universidade do Estado do Pará, Brasil E-mail: andrezaalves.p@ hotmail.com

Carla Suellem Sousa Araújo

ORCID: https://orcid.org/0000-0001-6844-4781 Universidade do Estado do Pará, Brasil

E-mail:carlaaraujoenfermagemuepa @ gmail.com

Maria Victoria Trindade de Sousa

ORCID: https://orcid.org/0000-0003-2034-361X

Universidade Federal do Oeste do Pará, Brasil

E-mail: mariaavic@icloud.com

Maria Jaqueline da Silva Portela

ORCID: https://orcid.org/0000-0002-4940-0326

Universidade Federal do Oeste do Pará, Brasil

E-mail: jaquelineportela.21.jp@gmail.com

Alda Lima Lemos

ORCID: https://orcid.org/0000-0001-5927-2535 Universidade do Estado do Pará, Brasil

E-mail: aldalimamoura@gmail.com

Ney Fonseca da Costa Junior

ORCID: https://orcid.org/0000-0002-5130-3242 Universidade do Estado do Pará, Brasil E-mail: ney.f.jr.96@gmail.com

Monique Cristine Prata Pinto

ORCID: https://orcid.org/0000-0002-8580-9647 Universidade do Estado do Pará, Brasil E-mail: moniqueprata@outlook.com

Fernanda da Silva Lima

ORCID: https://orcid.org/0000-0001-7067-4308 Universidade do Estado do Pará, Brasil E-mail: fernandalima_sl@outlook.com João Edivaldo da Silva Lobato

ORCID: https://orcid.org/0000-0001-5569-815X Universidade do Estado do Pará, Brasil

E-mail: ed2lobato@gmail.com

Larisse Oliveira Bezerra

ORCID: https://orcid.org/0000-0002-3109-4607 Universidade do Estado do Pará, Brasil

E-mail: larisseoliveirab@gmail.com

Daniela Cristina da Silva Maia

ORCID: https://orcid.org/0000-0002-8067-6953 Universidade do Estado do Pará, Brasil E-mail: mscdani@gmail.com

Thayana Pereira de Oliveira

ORCID: https://orcid.org/0000-0003-4526-9217

Escola Superior da Amazônia, Brasil

E-mail: thayanaenfa@gmail.com

Iza Belle Rodrigues Mendes

ORCID: https://orcid.org/0000-0003-3050-0156

Universidade Federal do Oeste do Pará, Brasil

E-mail: izabelle.rodrigues94@gmail.com 
Sayara Teixeira Potter da Rosa ORCID: https://orcid.org/0000-0001-9872-5728 Universidade da Amazônia, Brasil E-mail: sayarapotter@gmail.com

\begin{abstract}
Resumo
No contexto da saúde, diversos pontos devem ser levados em consideração para promover uma boa qualidade da assistência. É fundamental que os usuários tenham todas as informações sobre a condição de saúde que se encontram e, os profissionais que prestam serviços da assistência em saúde devem fornecer estas informações. Desta forma, busca-se identificar na literatura e descrever as tecnologias utilizadas para a educação em saúde na atenção primária em saúde. Trata-se de uma pesquisa exploratória, qualitativa, descritiva, do tipo revisão de literatura, cujo objeto de estudo foram artigos publicados de forma disponíveis na Biblioteca Virtual em Saúde. foram encontrados 60 artigos relacionados aos critérios de pesquisa e 9 publicações utilizadas para realização deste estudo que se enquadravam nos objetivos traçados. Identificou-se as tecnologias leve, leve-dura e dura utilizadas para educação em saúde. As tecnologias mais descritas são rodas de conversa, apresentação através de palestras, jogos dinâmicos, aplicativos interativos, além de sites com temáticas específicas para a promoção da saúde.
\end{abstract}

Palavras-chave: Educação em saúde; Atenção primária; Tecnologias em saúde.

\begin{abstract}
In the health context, several points must be taken into account to promote good quality of care. It is essential that users have all the information about the health condition they are in, and professionals who provide health care services must provide this information. Thus, we seek to identify in the literature and describe the technologies used for health education in primary health care. This is an exploratory, qualitative, descriptive, literature review, whose object of study were articles published in a form available in the Virtual Health Library. 60 articles were found related to the research criteria and 9 publications used to carry out this research. study that fit the outlined objectives. Light, light-hard and hard technologies used for health education were identified. The most described technologies are conversation wheels, presentation through lectures, dynamic games, interactive applications, in addition to websites with specific themes for health promotion.
\end{abstract}

Keywords: Health education; Primary care; Health technologies.

\title{
Resumen
}

En el contexto de la salud, se deben tener en cuenta varios puntos para promover una buena calidad de atención. Es fundamental que los usuarios tengan toda la información sobre el estado de salud en el que se encuentran, y los profesionales que prestan los servicios de salud deben proporcionar esta información. Así, buscamos identificar en la literatura y describir las tecnologías utilizadas para la educación en salud en la atención primaria de salud. Se trata de una revisión bibliográfica exploratoria, cualitativa, descriptiva, cuyo objeto de estudio fueron los artículos publicados en un formulario disponible en la Biblioteca Virtual en Salud. Se encontraron 60 artículos relacionados con los criterios de investigación y 9 publicaciones utilizadas para la realización de esta investigación. ajustarse a los objetivos trazados. Se identificaron tecnologías ligeras, ligeras y duras utilizadas para la educación sanitaria. Las tecnologías más descritas son ruedas de conversación, presentación a través de conferencias, juegos dinámicos, aplicaciones interactivas, además de sitios web con temas específicos para la promoción de la salud.

Palabras clave: Educación para la salud; Atención primaria; Tecnologías sanitarias.

\section{Introdução}

No contexto da saúde, diversos pontos devem ser levados em consideração para promover uma boa qualidade da assistência. É fundamental que os usuários tenham todas as informações sobre a condição de saúde que se encontram e, os profissionais que prestam serviços da assistência em saúde devem fornecer estas informações. Tais práticas promovem a autonomia do indivíduo e da coletividade e consequentemente qualidade na assistência. O conjunto de serviços prestados para o indivíduo e comunidade em prol da autonomia é caracterizado como Educação em Saúde (Seabra et al., 2019).

Para as práticas de Educação em Saúde os profissionais utilizam tecnologias que envolvem diversos materiais e métodos que auxiliam neste cuidado. Estas tecnologias podem ser classificadas em Leve, Leve-dura e Dura: sendo a primeira de baixa complexidade, de forma geral, é o acolhimento, gerenciamento, as relações interpessoais; a segunda de média complexidade, são as teorias de cuidado, materiais já estruturados; e a terceira de alta complexidade, são os equipamentos maquinários, normativas, estruturas organizacionais (Serrão, 2020; Sabino, et al., 2016). 
$\mathrm{Na}$ atenção primaria, o termo educação em saúde caracteriza-se em viabilizar a promoção da saúde, corroborando na prevenção de doenças, no tratamento precoce e eficaz das mesmas. Essas promoções, geralmente ocorrem através de ações voltadas a saúde juntamente com a comunidade, indivíduos ou grupos sociais permeando densamente as atividades que os profissionais de saúde realizam no âmbito das unidades, no domicílio, em outras instituições e nos espaços comunitários (Magalhães, 2018)

A educação em saúde se faz necessário como guia para a qualidade do cuidado, devendo estar sistematizada com base nos determinantes sociais da saúde, nas necessidades da população e de grupos vulneráveis, no perfil epidemiológico locorregional, com vivências teórico-práticas voltadas para a realidade desse grupo alvo (Machado \& Ximenes, 2018).

É necessário salientar a importância do profissional em saber identificar as problemáticas dos pacientes e quais necessitam de um trabalho em educação em saúde, levando em conta sempre a participação de uma equipe multidisciplinar, que sempre apresenta resultados positivos no que se relaciona as práticas assistenciais, pois além de se obter inúmeras alternativas no processo de saúde-doença, corrobora para um crescimento pessoal e profissional da equipe envolvida (Dias, Silveira \& Witt, 2009)

A busca pela interdisciplinaridade, é um processo constante de construção de relações para o enfrentamento aos novos desafios, para que ocorra é necessário unir os eixos a fim de compreender o processo saúde-doença. Segundo Farias e colaboradores (2017), trabalhar em equipe interprofissional significa atuar com profissionais de diversas formações na saúde, dispostos a transitar entre as áreas específicas de formação e de promover, além do ensino, a atuação interprofissional.

Neste contexto, são encontradas dificuldades para a execução da Educação em Saúde como falta de diálogo entre a equipe multiprofissional, dificuldades dos usuários em marcação de consultas, barreiras culturais, falta de recursos humanos e materiais, dentre outros (Andrade et al., 2016). Por isso, a melhoria na execução das redes de cuidados se faz necessária para uma estratégia mais eficaz, com treinamento e preparo da equipe atuante e o apoio informativo aos profissionais no que toca aos processos de mudanças, objetivando dinamizar as atividades desenvolvidas dentro da atenção primaria para atender de forma efetiva às necessidades dos usuários do sistema de saúde (Costa et al., 2014).

Considerando o exposto, traçou-se como objetivo identificar na literatura e descrever as tecnologias leve, leve-dura e dura utilizadas para a educação em saúde na atenção primária em saúde.

\section{Metodologia}

Trata-se de uma pesquisa exploratória, qualitativa, descritiva, do tipo revisão de literatura, realizada em março e abril de 2021. Foram incluídos na pesquisa artigos publicados em língua portuguesa, completos e disponíveis na plataforma Biblioteca Virtual da Saúde (BVS) nos anos de 2015 a 2021.

As bases de dados pesquisadas foram Literatura Latino-americana e do Caribe em Ciências da Saúde (LILACS), Base de Dados em Enfermagem (BDENF), Medical Literature Analysis and Retrieval System Online (MEDLINE), Bibliografia Brasileira de Odontologia (BBO), Idex Psicologia, Pan American Health Organization e Institutional Repository for Information Sharing (PAHO-IRIS), Conselho Nacional de Secretário de Saúde (CONASS), Secretaria de Saúde do Rio Grande do Sul (SES RS), Coleciona SUS, El Colegio Nacional (COLNAL) e Index Psicologia.

Utilizou-se para fazer o cruzamento de dados os Descritores em Ciências da Saúde (DeCS) Educação em Saúde + Atenção Primária + Tecnologias em Saúde. Para a mineração dos dados, aplicou-se um formulário contendo os objetivos traçados nesta pesquisa e, após selecionados, foram identificados nas publicações a compatibilidade com o tema. 


\section{Resultados}

De acordo com os descritores traçados, foram encontrados 60 artigos relacionados a pesquisa, sendo evidenciado de acordo com o Quadro 1 o quantitativo de publicação nos anos de 2015 a 2021.

Quadro 1 - Quantitativo dos artigos encontrados de acordo com o ano de publicação.

\begin{tabular}{|c|c|}
\hline ANO & N \\
\hline 2015 & 1 \\
\hline 2016 & 14 \\
\hline 2017 & 7 \\
\hline 2018 & 15 \\
\hline 2019 & 13 \\
\hline 2020 & 10 \\
\hline TOTAL & 60 \\
\hline
\end{tabular}

Fonte: Dados da pesquisa.

A distribuição dos artigos foi de 1 publicado no ano de 2015, 14 no ano de 2016, 7 no ano de 2017, 15 no ano de 2018, 13 em 2019 e 10 em 2020. Apesar de o ano de 2021 ser integrado na pesquisa, até o momento da captação dos dados, não foi encontrado nenhum artigo publicado neste ano

Com relação a base dedos, pode ser descrito de acordo com a Tabela 1.

Tabela 1 - Distribuição do quantitativo de artigos encontrados nas bases de dados de acordo com os critérios estabelecidos de pesquisa.

\begin{tabular}{|c|c|c|c|c|c|c|c|c|c|}
\hline \multirow{3}{*}{$\begin{array}{c}\text { Educação em Saúde + } \\
\text { Atenção Primária + } \\
\text { Tecnologias em saúde }\end{array}$} & \multicolumn{9}{|c|}{ BASE DE DADOS } \\
\hline & LILACS & BDENF & MEDLINE & $\begin{array}{l}\text { PAHO- } \\
\text { IRIS }\end{array}$ & $\begin{array}{c}\text { BDENF + } \\
\text { LILACS }\end{array}$ & $\begin{array}{c}\text { LILACS } \\
+ \\
\text { Index Psi }\end{array}$ & $\begin{array}{c}\text { LILACS } \\
+ \\
\text { BBO }\end{array}$ & $\begin{array}{c}\text { COLECIONA } \\
\text { SUS + } \\
\text { CONASS + } \\
\text { SES-RS }\end{array}$ & $\begin{array}{c}\text { LILACS } \\
+ \\
\text { BDENF } \\
+ \\
\text { COLNAL } \\
\end{array}$ \\
\hline & 34 & 3 & 5 & 1 & 10 & 1 & 3 & 1 & 3 \\
\hline TOTAL & \multicolumn{9}{|c|}{60} \\
\hline
\end{tabular}

Fonte: Dados da pesquisa.

Em LILACS foram encontrados 34 artigos, BDENF 3, MEDLINE 5 publicações, PAHO-IRIS 1, BDENF + LILACS 10 artigos, LILACS + Index Psicologia 1, LILACS + BBO 3 publicações, Coleciona SUS + CONASS + SES RS 1 artigo e LILACS + BDENF + COLNAL somente 3 artigos.

Das 60 publicações encontradas, 2 estavam duplicados e 49 não se enquadravam no presente estudo. Após a leitura dos artigos encontrados e a análise minuciosa das publicações, foram incluídas 9 publicações no presente estudo. Podendo ser descrito de acordo com a base de dados na Tabela 2. 
Tabela 2 - Distribuição do quantitativo dos artigos incluídos no estudo de acordo com as bases de dados.

\begin{tabular}{cccc}
\hline \multirow{2}{*}{ DESCRITOR } & \multicolumn{3}{c}{ BASE DE DADOS } \\
\cline { 2 - 4 } Incluídos & LILACS & BDENF & $\begin{array}{c}\text { BDENF + } \\
\text { LILACS }\end{array}$ \\
\cline { 2 - 4 } & 5 & 1 & 3 \\
\hline TOTAL & & 9 & \\
\hline
\end{tabular}

Fonte: Dados da pesquisa.

A partir do cruzamento dos descritores e a análise dos artigos, foram identificados para a pesquisa 9 publicações incluídas neste estudo. Sendo 5 presentes em LILACS, 1 em BDENF e 3 em LILACS + BDENF.

Com relação aos artigos incluídos no estudo e as identificações das bases de dados, são descritos no Quadro 2.

Quadro 2 - Distribuição dos artigos incluídos de acordo com autores, base de dados e ano de publicação.

\begin{tabular}{|c|c|c|c|c|}
\hline $\mathrm{N}$ & ARTIGO & AUTORES & BASE DE DADOS & $\begin{array}{c}\text { ANO DE } \\
\text { PUBLICAÇÃO }\end{array}$ \\
\hline A1 & $\begin{array}{l}\text { Implantação da puericultura e desafios do } \\
\text { cuidado na estratégia saúde da família em } \\
\text { um município do estado do Ceará }\end{array}$ & Soares et el. & LILACS & 2016 \\
\hline A2 & $\begin{array}{l}\text { Práticas de saúde na escola: um estudo } \\
\text { cartográfico na cidade de Parnaíba-PI }\end{array}$ & Melo \& Barros. & LILACS & 2016 \\
\hline A3 & $\begin{array}{c}\text { Fatores dificultadores na realização das } \\
\text { tecnologias leves no cuidado do } \\
\text { enfermeiro na atenção básica }\end{array}$ & Souza et al. & BDENF & 2018 \\
\hline A4 & $\begin{array}{c}\text { As atividades de grupo na perspectiva dos } \\
\text { sujeitos em uma Clínica da Família }\end{array}$ & Rego et al. & LILACS & 2019 \\
\hline A5 & $\begin{array}{l}\text { Validação de álbum seriado para } \\
\text { enfermeiros da atenção básica sobre } \\
\text { violência doméstica contra a mulher }\end{array}$ & Magalhães et al. & LILACS + BDENF & 2020 \\
\hline A6 & $\begin{array}{c}\text { Avaliação de objeto virtual de } \\
\text { aprendizagem sobre pré-natal para } \\
\text { adolescentes grávidas na atenção básica }\end{array}$ & Santiago et al. & LILCAS + BDENF & 2020 \\
\hline A7 & $\begin{array}{l}\text { Blogs e redes sociais na atenção à saúde } \\
\text { da família: o que a comunicação online } \\
\text { traz de novo? }\end{array}$ & $\begin{array}{l}\text { Barcelos, Lima \& } \\
\text { Aguiar. }\end{array}$ & LILACS & 2020 \\
\hline A8 & $\begin{array}{l}\text { Protocolo de atividade física remoto para } \\
\text { grupos de Academia da Saúde e } \\
\text { Estratégia de Saúde da Família }\end{array}$ & Novaes et al. & LILACS & 2020 \\
\hline A9 & $\begin{array}{l}\text { Elaboração e validação de um álbum } \\
\text { seriado para prevenção do pé diabético }\end{array}$ & Chaves et al & LILACS + BDENF & 2020 \\
\hline
\end{tabular}

Fonte: Dados da pesquisa.

Em relação aos objetivos e as descrições pertinentes a esta pesquisa são descritas abaixo no Quadro 3. 
Quadro 3 - distribuição das referências incluídas de acordo com objetivo, intervenção tecnológica, classificação das tecnologias e público alvo da intervenção.

\begin{tabular}{|c|c|c|c|c|}
\hline $\mathrm{N}$ & OBJETIVO & $\begin{array}{l}\text { INTERVENÇÃO } \\
\text { TECNOLÓGICA }\end{array}$ & $\begin{array}{c}\text { TIPO DE } \\
\text { TECNOLOGIA }\end{array}$ & PÚBLICO ALVO DA INTERVENÇÃO E CONCLUSÃO \\
\hline A1 & $\begin{array}{l}\text { Relatar a experiência da } \\
\text { implantação da puericultura e } \\
\text { desafios do cuidado na Estratégia } \\
\text { Saúde da Família em um município } \\
\text { do Estado do Ceará }\end{array}$ & $\begin{array}{l}\text { Acolhimento, } \\
\text { orientação, consulta } \\
\text { de enfermagem, } \\
\text { escuta qualificada, } \\
\text { visita domiciliar }\end{array}$ & $\begin{array}{l}\text { Leve e leve- } \\
\text { dura }\end{array}$ & $\begin{array}{l}\text { Prontuários de usuários da UBS. } \\
\text { Conclusão: Investiu-se nas ações de promoção da saúde, orientações } \\
\text { diversas quanto aos cuidados gerais, incentivo ao empoderamento dos } \\
\text { sujeitos envolvidos e estímulo a uma maior aproximação com o } \\
\text { serviço na perspectiva da saúde, e não somente diante de processos de } \\
\text { adoecimento. Para tanto, o fortalecimento do vínculo entre } \\
\text { profissionais de saúde e as famílias sob sua responsabilidade sanitária } \\
\text { possibilitou relações de confiança e respeito, percebendo-se uma } \\
\text { maior aceitação dos aprendizados proporcionados durante as consultas }\end{array}$ \\
\hline A2 & $\begin{array}{l}\text { Compreender concepções que } \\
\text { sustentam as práticas de saúde } \\
\text { desenvolvidas por profissionais de } \\
\text { saúde e educação no cotidiano da } \\
\text { escola, assim como discutir } \\
\text { processos de subjetivação } \\
\text { agenciados pelas práticas de saúde } \\
\text { no território escolar }\end{array}$ & $\begin{array}{l}\text { Acolhimento, } \\
\text { palestras, } \\
\text { orientações, } \\
\text { autonomização, } \\
\text { dinâmicas diversas }\end{array}$ & $\begin{array}{l}\text { Leve e leve- } \\
\text { dura }\end{array}$ & $\begin{array}{l}\text { sete professoras da lócus, o coordenador do PSE em Parnaíba, uma } \\
\text { enfermeira e uma agente comunitária de saúde da Estratégia de Saúde } \\
\text { da Família. } \\
\begin{array}{l}\text { Conclusão: Desse modo, abriu-se para a possibilidade de } \\
\text { desnaturalização das concepções de saúde, em que suas práticas } \\
\text { podem ir além da realização de atividades voltadas às práticas } \\
\text { normativas como higiene e alimentação, pensando-se em outros } \\
\text { modos de produzir saúde }\end{array}\end{array}$ \\
\hline A3 & \begin{tabular}{lcr} 
Identificar & os & \multicolumn{2}{c}{ obstáculos } \\
encontrados & na execução de \\
atividades & relacionadas & às \\
tecnologias & leves voltadas ao \\
cuidado do enfermeiro na Atenção \\
Básica
\end{tabular} & $\begin{array}{l}\text { Acolhimento, } \\
\text { promoção da saúde, } \\
\text { escuta qualificada, } \\
\text { atividades } \\
\text { educativas }\end{array}$ & $\begin{array}{l}\text { Leve e leve- } \\
\text { dura }\end{array}$ & $\begin{array}{l}15 \text { enfermeiros. } \\
\text { Conclusão: É imprescindível que exista o apoio da gestão oferecendo } \\
\text { ações de educação permanente para com os profissionais da saúde } \\
\text { sobre a importância da utilização das tecnologias interacionistas em } \\
\text { seus cotidianos de práticas }\end{array}$ \\
\hline A4 & $\begin{array}{l}\text { Analisar os sentidos que os sujeitos } \\
\text { conferem às atividades de grupos } \\
\text { realizadas em uma Clínica da } \\
\text { Família no Rio de Janeiro }\end{array}$ & $\begin{array}{l}\text { Acolhimento, } \\
\text { orientações, } \\
\text { palestra, dinâmicas } \\
\text { em grupo, artesanato }\end{array}$ & $\begin{array}{l}\text { Leve e leve- } \\
\text { dura }\end{array}$ & $\begin{array}{l}21 \text { usuários da unidade básica de saúde. } \\
\text { Conclusão: Os resultados demonstram que as abordagens de grupo } \\
\text { podem contribuir com a transformação do modelo hegemônico } \\
\text { biomédico das práticas de saúde }\end{array}$ \\
\hline A5 & $\begin{array}{l}\text { Validar o conteúdo e a aparência de } \\
\text { um álbum seriado para enfermeiros } \\
\text { da atenção básica sobre violência } \\
\text { doméstica contra a mulher }\end{array}$ & Álbum seriado & Leve-dura & $\begin{array}{l}15 \text { especialistas. } \\
\text { Conclusão: o álbum seriado foi validado quanto ao conteúdo e } \\
\text { aparência, sugerindo que esta tecnologia educativa possibilita ações } \\
\text { de educação em saúde realizadas por enfermeiros da Atenção Básica }\end{array}$ \\
\hline A6 & $\begin{array}{l}\text { Descrever o processo de construção } \\
\text { e avaliação de um Objeto Virtual } \\
\text { de Aprendizagem sobre pré-natal } \\
\text { para adolescentes grávidas no } \\
\text { contexto da atenção básica }\end{array}$ & $\begin{array}{l}\text { Objetos Virtuais de } \\
\text { Aprendizagem }\end{array}$ & Dura & $\begin{array}{l}88 \text { adolescentes grávidas. } \\
\text { Conclusão: Acredita-se que o GESTAQ possa ser usado como } \\
\text { recurso digital complementar ao processo educativo de adolescentes } \\
\text { grávidas no contexto da atenção básica para fornecer orientações } \\
\text { sobre pré-natal }\end{array}$ \\
\hline A7 & $\begin{array}{l}\text { O estudo que fundamenta este } \\
\text { artigo investiga a criação de } \\
\text { ambientes de comunicação online } \\
\text { nos serviços de atenção primária no } \\
\text { município do Rio de Janeiro, } \\
\text { selecionando o caso do } \\
\text { Observatório de Tecnologia de } \\
\text { Informação e Comunicação em } \\
\text { Sistemas e Serviços de Saúde } \\
\text { (Otics - Rio), posteriormente } \\
\text { substituído pelos Ambientes de } \\
\text { Saberes e Aprendizagens em Saúde } \\
\text { (Asas - Rio) }\end{array}$ & Blogs & Dura & $\begin{array}{l}\text { Rastreamento virtual. } \\
\text { Conclusão: Observou-se que a comunicação estabelecida mantém } \\
\text { uma tendência difusionista, característica do modelo informacional de } \\
\text { comunicação, especialmente nos blogs, evidenciando que é mesmo } \\
\text { possível tender à reprodução de antigos modelos e sentidos de } \\
\text { comunicação e saúde }\end{array}$ \\
\hline A8 & $\begin{array}{l}\text { o objetivo deste estudo foi } \\
\text { apresentar o modelo lógico para } \\
\text { elaboração e implementação de um } \\
\text { protocolo de Prática } \\
\text { Corporal/Atividade Física remota, } \\
\text { disponibilizado por tecnologias, e } \\
\text { avaliar a adesão dos usuários a este } \\
\text { protocolo }\end{array}$ & $\begin{array}{l}\text { Aplicativos } \quad \text { de } \\
\text { mensagens, vídeos } \\
\text { educativos, reuniões } \\
\text { virtuais }\end{array}$ & Dura & $\begin{array}{l}754 \text { usuários. } \\
\text { Conclusão: No caso dos usuários que estão trabalhando em casa, } \\
\text { também há a orientação de tentar fazer pausas regulares durante o dia } \\
\text { para se movimentar e/ou passear pela casa durante chamadas em } \\
\text { conferência. Até o presente momento, os PEF não conseguiram que os } \\
\text { usuários registrem suas rotinas de exercícios físicos e, por isso, não se } \\
\text { pode afirmar que os usuários estão atingindo os } 150 \mathrm{~min} / \mathrm{semana} \\
\text { recomendados pela OMS }\end{array}$ \\
\hline A9 & $\begin{array}{l}\text { elaborar e validar o conteúdo e } \\
\text { aparência de um álbum seriado } \\
\text { sobre prevenção do pé diabético } \\
\text { para utilização por profissionais da } \\
\text { Atenção Primária à Saúde }\end{array}$ & Álbum seriado & Leve-dura & $\begin{array}{l}\text { sete especialistas com experiência em educação em saúde. } \\
\text { Conclusão: o álbum seriado foi considerado válido pelos especialistas } \\
\text { e uma tecnologia capaz de auxiliar o profissional de saúde no rastreio } \\
\text { do pé em risco da pessoa com diabetes mellitus }\end{array}$ \\
\hline
\end{tabular}

Fonte: Dados da pesquisa. 


\title{
4. Discussão
}

Segundo Barbosa (2019) as tecnologias podem ser divindades em três categorias:

\begin{abstract}
“A leve refere-se às tecnologias de relações do tipo produção de vínculo, autonomização, acolhimento, gestão como uma forma de governar processos de trabalho. A leve-dura diz respeito aos saberes estruturados, que operam no processo de trabalho em saúde como a clínica médica, clínica psicanalítica, a epidemiologia, o taylorismo e o fayolismo. A dura é referente ao uso de equipamentos tecnológicos do tipo máquinas, normas e estruturas organizacionais".
\end{abstract}

A partir de agora iremos relacionar os artigos selecionados a partir do método de pesquisa definido divididos nessas três categorias.

\subsection{Tecnologias Leves}

As tecnologias leves condensam em si as relações de interação e subjetividade. Elas possibilitam a produção do acolhimento, vínculo, responsabilização e autonomização do indivíduo. A partir disso, dentre os artigos selecionados, 4 deles fazem o uso da tecnologia leve para a promoção da educação em saúde na atenção básica (Sabino, 2016).

Soares (2016) fez uso de ações de promoção de saúde durante as consultas de pré-natal com orientações diversas quanto aos cuidados gerais e com as crianças, como o incentivo ao aleitamento materno exclusivo até os seis meses de idade. Para ser feita uma maior aproximação com a realidade da mãe e suas particularidades, foram realizadas visitas domiciliares durante o período puerperal. Esta visita é recomendada pelo Ministério da Saúde (MS) durante a primeira semana após o parto.

Para a construção de um relacionamento harmonioso entre mãe e filho e o profissional de saúde deve-se haver a promoção do cuidado através do diálogo, do acolhimento e interação entre ambos, sendo que isto pode ser feito a partir de tecnologias simples e de forma fácil durante uma consulta de enfermagem ou visita domiciliar a partir de escuta qualificada (Queiroz, 2006).

Barros (2016) também fez uso de tecnologias leves durante sua pesquisa-intervenção em uma escola de educação infantil da cidade de Parnaíba-PI onde era realizado o estágio prático de Saúde Coletiva. Durante esse período foi feito o acolhimento, palestras, orientações, autonomização, dinâmicas diversas com os profissionais do ambiente escolar para conhecer as suas concepções que sustentam as práticas de saúde desenvolvidas por profissionais de saúde e educação no cotidiano da escola.

No entanto, durante esse processo de diálogo com os profissionais foi possível verificar algumas lacunas que não tornam a atuação de modo efetivo. Como por exemplo uma das atividades realizadas como a verificação do cartão de vacina das crianças da escola estava voltada, sobretudo, à vigilância epidemiológica e se reduzia a medir, pesar, verificação do cartão e preenchimento do cadastro. O que não tornava o processo um momento de reconhecimento das condições de saúde de cada criança a partir de suas particularidades.

As tecnologias leves são as que possibilitam captar a singularidade, o contexto, o universo cultural, os modos específicos de viver determinadas situações por parte do usuário (Santos, 2017). No estudo de Alves (2018) foi procurado identificar fatores que dificultam a realização de tecnologias leves no cuidado do enfermeiro na atenção básica, a partir de uma entrevista semiestruturada com 15 enfermeiros que atuavam na Estratégia de Saúde da Família do município de Cajazeiras, Paraíba ele notou que o tempo reduzido de atendimento à população, a estrutura física precária, a alta demanda de trabalho impediam de haver um contato eficaz e de acolhimento com o paciente.

Por último, Baião (2019) analisou os sentidos que os sujeitos conferem às atividades de grupos realizadas em uma Clínica da Família no Rio de Janeiro, ao todo foram 21 participantes desses grupos. Nesses grupos a intervenção tecnológica 
aplicada era o acolhimento, orientações, palestras e dinâmicas em grupos. Os resultados demonstram que as abordagens de grupo podem contribuir com a transformação do modelo hegemônico biomédico das práticas de saúde.

\subsection{Tecnologias Leve-Dura}

Dois artigos abordaram o uso da tecnologia leve-dura. Almeida (2020) elaborou um álbum seriado para enfermeiros da atenção básica com a temática sobre a violência doméstica contra a mulher. Este material foi elaborado com o intuito de ter seu conteúdo validado por especialistas pré-selecionados. O álbum seriado foi validado quanto ao conteúdo e aparência, sugerindo que esta tecnologia educativa possibilita ações de educação em saúde realizadas por enfermeiros da Atenção Básica.

Relacionado ao estudo anterior, Chaves (2021) também desenvolveu uma metodologia semelhante, porém o público alvo do álbum seriado seria para pacientes com diabetes mellitus (DM), onde seria abordado o tema a para prevenção do pé diabético a ser também utilizado por profissionais da atenção básica. O álbum seriado foi considerado válido pelos especialistas e uma tecnologia capaz de auxiliar o profissional de saúde no rastreio do pé em risco da pessoa com diabetes mellitus.

Barbosa (2019) diz que a utilização da tecnologia leve-dura pode colocar a pessoa que participa da prática na posição de protagonista do seu processo de aprendizagem, quando este coloca em prática o que aprende, somado aos seus conhecimentos adquiridos durante o cotidiano. Os jogos são exemplos de momentos de descontração, mas também de assimilação de conhecimento.

Além disso, a tecnologia leve-dura ultrapassa a compreensão de conhecimentos tecnológicos estruturados e apresenta liberdade em que os profissionais tem opções de escolha de suas ações, sendo assim, o trabalho em saúde é permeado de subjetividades, as quais são atreladas de acordo àqueles que são participantes das interações (Sabino, 2016).

\subsection{Tecnologias Dura}

As tecnologias duras podem ser definidas como aquelas que são feitas por equipamentos, instrumentais e máquinas utilizados para os procedimentos executados por profissionais ou de forma configurada (Santiago, 2019). Em relação ao uso das tecnologias duras, Andrade (2019) procurou descrever o processo de construção e avaliação de um Objeto Virtual de Aprendizagem sobre pré-natal para adolescentes grávidas no contexto da atenção básica denominado de GESTAQ. Acredita-se que o GESTAQ possa ser usado como recurso digital complementar ao processo educativo de adolescentes grávidas no contexto da atenção básica para fornecer orientações sobre pré-natal.

Aguiar (2020) investigou a criação de ambientes de comunicação online nos serviços de atenção primária no município do Rio de Janeiro, selecionando o caso do Observatório de Tecnologia de Informação e Comunicação em Sistemas e Serviços de Saúde (Otics - Rio), posteriormente substituído pelos Ambientes de Saberes e Aprendizagens em Saúde (Asas Rio). Observou-se que a comunicação estabelecida mantém uma tendência difusionista, característica do modelo informacional de comunicação, especialmente nos blogs, evidenciando que é mesmo possível tender à reprodução de antigos modelos e sentidos de comunicação e saúde.

Alves (2020) apresentou o modelo lógico para elaboração e implementação de um protocolo de Prática Corporal/Atividade Física remota, disponibilizado por tecnologias, e avaliar a adesão dos usuários a este protocolo, que foram 754 usuários. No caso dos usuários que estão trabalhando em casa, também há a orientação de tentar fazer pausas regulares durante o dia para se movimentar e/ou passear pela casa durante chamadas em conferência. Até o presente momento, os PEF não conseguiram que os usuários registrem suas rotinas de exercícios físicos e, por isso, não se pode afirmar que os usuários estão atingindo os $150 \mathrm{~min} / \mathrm{semana}$ recomendados pela OMS. Além disso, os usuários mais idosos relataram terem dificuldade de acesso a esses recursos tecnológicos. 


\section{Conclusão}

A procura por métodos e técnicas de educação em saúde deve perpassar todos os níveis de assistência, visando, além da prevenção dos cuidados, na atenção primária, a continuidade da assistência nas atenções secundária e terciária. As instituições de saúde bem como os profissionais devem adotar práticas educativas para proporcionar recuperação do paciente e elucidar temáticas indispensáveis para a assistência.

Desta forma, as tecnologias em saúde são de extrema importância para subsidiar uma assistência de qualidade, uma vez que a educação em saúde faz parte da continuidade do cuidado e proporciona maior autonomia para a promoção em saúde. Tais ferramentas são responsáveis por auxiliar os profissionais tanto para capacitação da equipe em saúde como para promover assistência para a população. É necessário que sejam realizados estudos voltados para a qualificação dos profissionais que utilizam estas ferramentas tecnológicas e para a procura por mais ferramentas que possam ser adequadas de acordo com a realidade da assistência da população.

A partir do estudo realizado evidencia-se a importância da temática para a comunidade acadêmica e científica, bem como para profissionais da saúde, isto posto, sugere-se pesquisas que contribuam para a criação de novas tecnologias em saúde visando a melhoria da qualidade da assistência em saúde.

\section{Referências}

Andrade, R. S. D., Caldas, L. B. S. D. N., Falcão, M. L. P., \& Góes, P. S. A. D. (2016). Processo de trabalho em unidade de saúde da família e a educação permanente. Trabalho, Educação e Saúde, 14, 505-521.

Barcelos, P. E. L., Lima, T. V., \& de Aguiar, A. C. (2020). Blogs e redes sociais na atenção à saúde da família: o que a comunicação online traz de novo? Revista Eletrônica de Comunicação, Informação e Inovação em Saúde, 14(1).

Costa, J. P., Jorge, M. S. B., Vasconcelos, M. G. F., Paula, M. L. D., \& Bezerra, I. C. (2014). Resolubilidade do cuidado na atenção primária: articulação multiprofissional e rede de serviços. Saúde em debate, 38, 733-743.

Chaves, M. A. A., Santos, R. F. D., Moura, L. K. B., Lago, E. C., Sousa, K. H. J. F., \& Almeida, C. A. P. L. (2021). Elaboração e validação de um álbum seriado para prevenção do pé diabético. Revista Cuidarte, 12(1).

Dias, V. P., Silveira, D. T., \& Witt, R. R. (2009). Educação em saúde: protocolo para o trabalho de grupos em Atenção Primária à Saúde. Revista de APS, 12(2)

Farias, D. N. D., Ribeiro, K. S. Q. S., Anjos, U. U. D., \& Brito, G. E. G. D. (2017). Interdisciplinaridade e interprofissionalidade na estratégia saúde da família. Trabalho, Educação e Saúde, 16, 141-162.

Machado, M. H., \& Ximenes, F. R. G. (2018). Gestão da Educação e do Trabalho em Saúde no SUS: trinta anos de avanços e desafios. Ciência \& Saúde Coletiva, 23, 1971-1979.

Magalhães, V. M. D. P. R., dos Santos, R. F., Ramos, C. V., Feitosa, L. G. G. C., Lago, E. C., Sousa, E. K. S., \& Almeida, C. A. P. L. (2020). Validação de álbum seriado para enfermeiros da atenção básica sobre violência doméstica contra a mulher. Cogitare Enfermagem, 25.

Magalhães, R. (2018). Governança, redes sociais e promoção da saúde: reconfigurando práticas e institucionalidades. Ciência \& Saúde Coletiva, 23, 31433150 .

Melo, A. C. V., \& Barros, J. P. P. (2016). Práticas de saúde na escola: um estudo cartográfico na cidade de Parnaíba-PI. Revista Pesquisas e Práticas Psicossociais, 11(2), 341-355.

Moraes de Sabino, L. M., Rabelo Magalhães Brasil, D., Áfio Caetano, J., Lavinas Santos, M. C., \& Santos Alves, M. D. (2016). Uso de tecnologia leve-dura nas práticas de enfermagem: análise de conceito. Aquichan, 16(2), 230-239.

Nóbrega Novaes, C. R. M., Wanderley, F. A. C., Falcão, I. M., Alves, R. B., Lima, A. T., \& Soares, M. C. B. (2020). Protocolo de atividade física remoto para grupos de Academia da Saúde e Estratégia de Saúde da Família. Revista Brasileira de Atividade Física \& Saúde, 25, 1-6.

Queiroz, M. V., \& Jorge, M. S. (2006). Estratégias de educação em saúde e a qualidade do cuidar e ensinar em pediatria: a interação, o vínculo e a confiança no discurso dos profissionais. Interface-Comunicação, Saúde, Educação, 10, 117-130.

Rego, L. S., Figueiredo, G. D. O., Romano, V. F., \& Baião, M. R. (2019). As atividades de grupo na perspectiva dos sujeitos em uma Clínica da Família. Physis: Revista de Saúde Coletiva, 29, e290316.

Rodrigues, V. E. S., de Sousa Neves, I., da Silva Siqueira, I. H., de Araujo Pereira, M. K., Araújo, S. N. M., Bastos, N., \& de Oliveira, A. S. S. (2019). Aplicabilidade de tecnologias leve-duras como estratégia para cuidadores de idosos: relato de experiência. Revista Enfermagem Digital Cuidado e Promoção da Saúde, 4, 2. 
Research, Society and Development, v. 10, n. 16, e255101623909, 2021

(CC BY 4.0) | ISSN 2525-3409 | DOI: http://dx.doi.org/10.33448/rsd-v10i16.23909

Rosário Serrão, T. (2020). O papel da tecnologia leve no processo de gestão em enfermagem no setor de urgência e emergência. Biodiversidade, 19(4).

Santiago, R. F., Andrade, E. M. L. R., Mendes, I. A. C., Viana, M. C. A., \& Nery, I. S. (2020). Avaliação de objeto virtual de aprendizagem sobre pré-natal para adolescentes grávidas na atenção básica. Acta Paulista de Enfermagem, 33.

Santos, L. F. O., de Almeida Sebastião, M. L. V., Franco, R. O. M., Martins, M. L., da Silva, C. D. P. O., \& de Souza, E. R. P. (2017). Desmistificação da simplicidade das tecnologias leves no processo de trabalho em medicina de família e comunidade. Revista de Saúde, 8(1 S1), 123 -124.

Souza, J. W. R. D., Silva, F. C. V., Brito, P. K. H., Silva, R. C. R. D., Alves, B., \& Fernandes, M. C. (2018). Fatores dificultadores na realização das tecnologias leves no cuidado do enfermeiro na atenção bá-sica. Rev. enferm. atenção saúde, 63-75.

Souza, F. M. D. L. C., dos Santos, W. N., da Costa Santos, R. S., Rodrigues, O. B., Santiago, J. D. C. D., \& da Silva, R. A. R. (2019). Tecnologias apropriadas ao processo do trabalho de parto humanizado. Enfermagem em Foco, 10(2).

Seabra, C. A. M., Xavier, S. P. L., Sampaio, Y. P. C. C., Oliveira, M. F. D., Quirino, G. D. S., \& Machado, M. D. F. A. S. (2019). Educação em saúde como estratégia para promoção da saúde dos idosos: Uma revisão integrativa. Revista Brasileira de Geriatria e Gerontologia, 22.

Soares, D. G., Pinheiro, M. C. X., de Queiroz, D. M., \& Soares, D. G. (2016). Implementation of childcare and care challenges in the family health strategy in a city of the Ceará state. Revista Brasileira em Promocao da Saude, 29(1), 132. 